\title{
Macroscopic conservation equation based model for surface tension driven flow
}

\author{
T. M. Adams \& A. R. White \\ Department of Mechanical Engineering, \\ Rose-Hulman Institute of Technology, USA
}

\begin{abstract}
Due to the small scales encountered in MEMS and microfluidic devices, surface tension can play a significant role in the device physics. As such, many microscale devices have exploited surface tension forces to serve as passive valves and to move fluids. Surprisingly, few models for surface tension driven flow seem to have been developed.

In this study a theoretical mathematical model for surface tension driven flow through a capillary of an arbitrary cross section is derived. The model is based on macroscopic mass and momentum balances, allowing for different physical insights to be drawn compared to approaches starting with the Navier-Stokes equations. Expressions for flow length and velocity are derived for both frictionless flow and for flow with wall friction. For frictionless flow the result of surface tension force is to increase the momentum of the fluid by constantly drawing mass into the capillary at a constant characteristic velocity. In the case of flow with wall friction, a characteristic time scale can be calculated. For times on the order of the characteristic time scale, the flow velocity exponentially decays from the characteristic velocity, whereas for larger times the velocity shows an inverse power law dependence. The model well predicts flow behaviour for previously published experiments in which the characteristic time is small. For systems in which the characteristic time is larger, the model may show improved performance over previous models.
\end{abstract}

Keywords: capillary, microchannel, microfluidics, surface tension.

\section{Introduction}

With the rapid development of microfluidic devices over the past decade, the favourable scaling of surface tension with small dimensions has been exploited 
for many purposes, including for use as passive valves [1], [2] and the seemingly ubiquitous capillary electrophoresis [3], [4] used as chemical and biological detection methods. Surprisingly, few models seem to be available to address the physics of liquid flows driven by surface tension.

Studies on the nature of interfacial surface tension and the dynamics of capillary wetting date back to over a century ago, including the early works by Young [5], Lucas [6], and Washburn [7]. More recently, the methods and equations developed by these early studies have been modified for use in parallel plate geometries by Schwiebert and Leong [8] and Jong et al. [9]. These recent studies have shown good agreement between predictions and experimentally obtained parameters.

The method of analysis in the above mentioned studies has typically started with a quasi-steady version of the Navier-Stokes equations and then introduced the non-steady effect of surface tension via manipulation of the applied pressure gradient term. Furthermore, the works focus on particular geometries and lack a general applicability to other capillary shapes.

The current study investigates surface tension driven flow in capillaries of arbitrary cross section. Rather than starting with differential equations of motion, the current study uses macroscopic conservation laws applied to the entire capillary in order to model the flow. The results of the model are compared to previous models, as well as the experimental predictions therein.

\section{Frictionless flow model}

As a starting point for modelling surface tension driven flow we begin with a frictionless flow model.

\subsection{Conservation of mass}

Figure 1 gives a schematic diagram of the open system used in the macroscopic mass conservation equation. A reservoir feeds flow into a capillary of arbitrary cross section with perimeter $\mathcal{P}$ and cross sectional area $A_{c}$. The leftmost boundary of the system remains stationary at the capillary inlet whereas the rightmost boundary moves with the liquid-gas interface at a distance $L$ from the inlet.

Conservation of mass for this system requires

$$
\frac{d m_{s y s}}{d t}=\dot{m}_{i n},
$$

where $m_{s y s}$ is the mass contained in the system at any time $t$ and $\dot{m}_{i n}$ is the rate of mass flow into the system. Eq. (1) reduces to

$$
\frac{d\left(\rho A_{c} L\right)}{d t}=\rho A_{c} V
$$

where $\rho$ is density and $V$ is the average velocity of the fluid at the capillary inlet. For an incompressible fluid, $\rho$ is constant and Eq. (2) reduces to

$$
V=\frac{d L}{d t}
$$




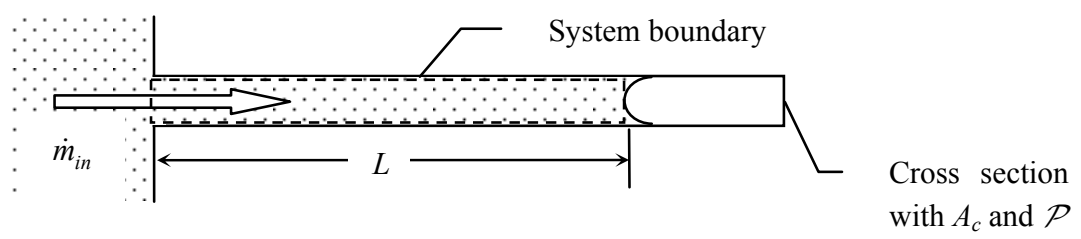

Figure 1: Schematic diagram of system used in macroscopic mass conservation.

The analysis would be identical for a system with a stationary inlet at any location along the capillary. Thus, at any point in time the entire fluid in the capillary moves with a single velocity equal to velocity of the liquid-gas interface.

\subsection{Conservation of linear momentum}

Figure 2 shows the open system used in the macroscopic momentum conservation equation. The leftmost boundary of the system extends just inside the reservoir where pressure is constant and the fluid velocity is approximately zero. The rightmost boundary coincides with the liquid-gas interface.

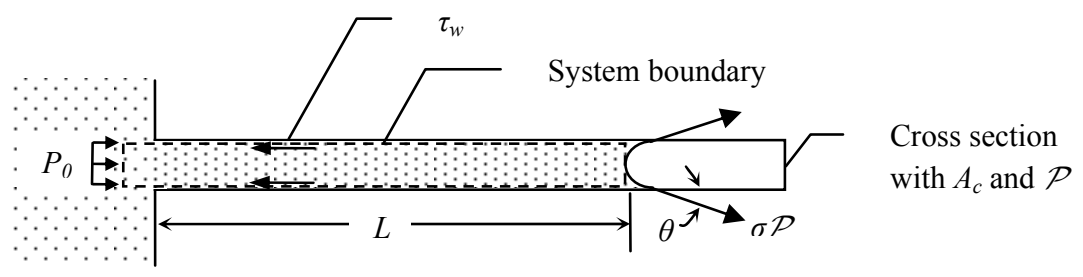

Figure 2: Schematic diagram of system used in macroscopic momentum conservation.

The flow direction component of linear momentum conservation requires that

$$
\frac{d(m V)_{\text {sys }}}{d t}=\sum F_{\text {external }} \text {. }
$$

For frictionless flow the wall shear stress, $\tau_{w}$ in Fig.2 is zero and Eq. (4) becomes

$$
\frac{d(m V)}{d t}=P_{0} A_{c}+\sigma \cos \theta \mathcal{P},
$$

where $P_{0}$ is gage pressure in the reservoir, $\sigma$ is surface tension and $\theta$ is the contact angle formed by the liquid-gas interface with the capillary wall. Integrating with respect to time yields

$$
m V=\left(P_{0} A_{c}+\sigma \cos \theta \mathcal{P}\right) t+c_{1} .
$$


At $t=0$ the momentum of the system is zero, so that the constant of integration is $c_{1}=0$.

From the conservation of mass results, $V=d L / d t$. Substituting this along with $m=\rho A_{c} L$ into Eq. (6) gives

$$
\rho A_{c} L \frac{d L}{d t}=\left(P_{0} A_{c}+\sigma \cos \theta \mathcal{P}\right) t .
$$

Separating variables, integrating and solving for $L$,

$$
L=\left(\left(\frac{P_{0}}{\rho}+\frac{\sigma \cos \theta \mathcal{P}}{\rho A_{c}}\right) t^{2}+c_{2}\right)^{1 / 2} .
$$

At $t=0, L=0$, so that the constant of integration is $c_{2}=0$.

Eq. (8) can be differentiated with respect to $t$ to find $V$; equivalently, Eq. (8) can be substituted into Eq. (7), which in turn can be solved for velocity. Either way the result is

$$
V=\frac{d L}{d t}=\left(\frac{P_{0}}{\rho}+\frac{4 \sigma \cos \theta}{\rho D_{h}}\right)^{1 / 2}
$$

where $P$ and $A_{c}$ have been eliminated in favour of the hydraulic diameter,

$$
D_{h} \equiv \frac{4 A_{c}}{\mathcal{P}} .
$$

For the case in which $P_{0}=0$, corresponding to a reservoir height of zero, the flow is driven completely by surface tension. Eq. (9) gives a characteristic velocity for this situation, one that represents a theoretical upper limit for flow velocity.

$$
V_{c h r}=\left(\frac{4 \sigma \cos \theta}{\rho D_{h}}\right)^{1 / 2}
$$

This velocity can also be found by setting the Weber number based on hydraulic diameter equal to $4 \cos \theta$ :

$$
W e \equiv \frac{\rho V_{c h r}^{2} D_{h}}{\sigma}=4 \cos \theta
$$

It is of interest to note that the flow velocity given by either Eq. (9) or Eq. (11) is constant. Therefore, the result of the surface tension force is to increase the system momentum, not by increasing its velocity, but by constantly drawing more mass into the capillary.

\section{Flow model with wall friction}

Due to the large surface area to volume ratios encountered in systems in which surface tension is important, the assumption of zero wall friction, a surface phenomenon, is not realistic. Though the frictionless model yielded a theoretical upper limit for flow velocity, in practice we would expect to see slower velocities due to the impeding effect of wall shear stress. Thus, a more accurate model should include the effects of wall friction. 


\subsection{Conservation of mass}

As the presence of wall shear stress does not affect any of the terms appearing in the conservation of mass equation, the analysis of section 2.1 is still valid. At any point in time the entire fluid in the capillary again moves with a single velocity equal to velocity of the liquid-gas interface as given in Eq. (3).

\subsection{Conservation of linear momentum}

The flow direction component of linear momentum conservation given by Eq. (4) as applied to the system in Fig.2 including wall friction is

$$
\frac{d(m V)}{d t}=P_{0} A_{c}-\tau_{w} \mathcal{P} L+\sigma \cos \theta \mathcal{P} .
$$

The wall shear stress, $\tau_{w}$, is related to the Fanning friction factor, $c_{f}$, by

$$
\tau_{w}=c_{f} \frac{1}{2} \rho V^{2} .
$$

Assuming that the flow is fully developed at any point in time, a reasonable assumption given the small entrance lengths encountered in microfluidics, the Fanning friction factor is given by

$$
c_{f}=\frac{C}{R e},
$$

where $R e$ is the Reynolds number based on the hydraulic diameter and $C$ is a constant depending solely on the geometry of the cross section. Using Eqs. (14) and (15), along with the definition of Reynolds number,

$$
R e=\frac{\rho V D_{h}}{\eta},
$$

Eq. (13) becomes

$$
\frac{d(m V)}{d t}=P_{0} A_{c}-\frac{C \eta}{\rho V D_{h}}\left(\frac{1}{2} \rho V^{2}\right) \mathcal{P} L+\sigma \cos \theta \mathcal{P} .
$$

Recognizing that $m=\rho A_{c} L$ allows Eq. (17) to be rearranged as

$$
\frac{d(m V)}{d t}=P_{0} A_{c}-\frac{2 C \eta}{\rho D_{h}^{2}}(m V)+\sigma \cos \theta \mathcal{P} .
$$

Separating variables,

$$
\frac{1}{P_{0} A_{c}-\frac{2 C \eta}{\rho D_{h}^{2}}(m V)+\sigma \cos \theta \mathcal{P}} d(m V)=d t .
$$

Integrating and solving for $m V$,

$$
m V=t_{c h r}\left[P_{0} A_{c}+\sigma \cos \theta \mathcal{P}-c_{1} \exp \left(-t / t_{c h r}\right)\right],
$$

where $c_{1}$ is a constant of integration and $t_{c h r}$ is a characteristic time of the system given by

$$
t_{c h r}=\frac{\rho D_{h}^{2}}{2 C \eta} .
$$


At $t=0$ the momentum of the system is zero, so that the constant of integration is found to be

$$
c_{1}=P_{0} A_{c}+\sigma \cos \theta \mathcal{P}
$$

Eq. (20) is now

$$
m V=t_{c h r}\left(P_{0} A_{c}+\sigma \cos \theta \mathcal{P}\right)\left[1-\exp \left(-t / t_{c h r}\right)\right] .
$$

As was the case with the frictionless flow model, the length $L$ can be found by substituting $V=d L / d t$ and $m=\rho A_{c} L$ into the expression above.

$$
\rho A_{c} L \frac{d L}{d t}=t_{c h r}\left(P_{0} A_{c}+\sigma \cos \theta \mathcal{P}\right)\left[1-\exp \left(-t / t_{c h r}\right)\right]
$$

Separating variables, integrating and solving for $L$ yields

$$
L=\left\{2\left[t_{c h r}\left(\frac{P_{0}}{\rho}+\frac{4 \sigma \cos \theta}{\rho D_{h}}\right)\left(t+t_{c h r} \exp \left(-t / t_{c h r}\right)\right)+c_{2}\right]\right\}^{1 / 2},
$$

where we have once again eliminated $\mathcal{P}$ and $A_{c}$ for $D_{h}$. Requiring that $L=0$ at $t=0$ yields the constant of integration, $c_{2}$.

$$
c_{2}=-t_{c h r}^{2}\left(\frac{P_{0}}{\rho}+\frac{4 \sigma \cos \theta}{\rho D_{h}}\right)
$$

Eq. (25) becomes

$$
L=\left\{2 t_{c h r}\left(\frac{P_{0}}{\rho}+\frac{4 \sigma \cos \theta}{\rho D_{h}}\right)\left(t-t_{c h r}\left[1-\exp \left(-t / t_{c h r}\right)\right]\right)\right\}^{1 / 2} .
$$

If we again let $P_{0}=0$ and focus on surface tension driven flow only, this reduces to

$$
L=\left\{\frac{4 \sigma \cos \theta}{\rho D_{h}} 2 t_{c h r}\left(t-t_{c h r}\left[1-\exp \left(-t / t_{c h r}\right)\right]\right)\right\}^{1 / 2} .
$$

The reader will recognize the characteristic velocity of Eq. (11) appearing in Eq. (28), which can be recast as

$$
L=V_{c h r}\left(2 t_{c h r}\left(t-t_{c h r}\left[1-\exp \left(-t / t_{c h r}\right)\right]\right)\right)^{1 / 2} .
$$

As before the flow velocity can be found simply by differentiating the length with respect to time. The result is

$$
V=V_{c h r} \frac{\sqrt{2 t_{c h r}}}{2} \frac{1-\exp \left(-t / t_{c h r}\right)}{\left[t-t_{c h r}\left(1-\exp \left(-t / t_{c h r}\right)\right)\right]^{1 / 2}} .
$$

Both the length and velocity as given by Eqs. (29) and (30) initially show an exponential decay. For times much larger than the characteristic time scale $t_{c h r}$, the exponential terms can be neglected, and Eqs. (29) and (30) respectively reduce to 


$$
L=V_{c h r}\left(2 t_{c h r} t\right)^{1 / 2}
$$

and

$$
V=V_{c h r}\left(\frac{t_{c h r}}{2 t}\right)^{1 / 2} .
$$

And so for times larger than the characteristic time, both length and velocity show a power law dependence on time. Figures 3 and 4 illustrate these trends in dimensionless form.

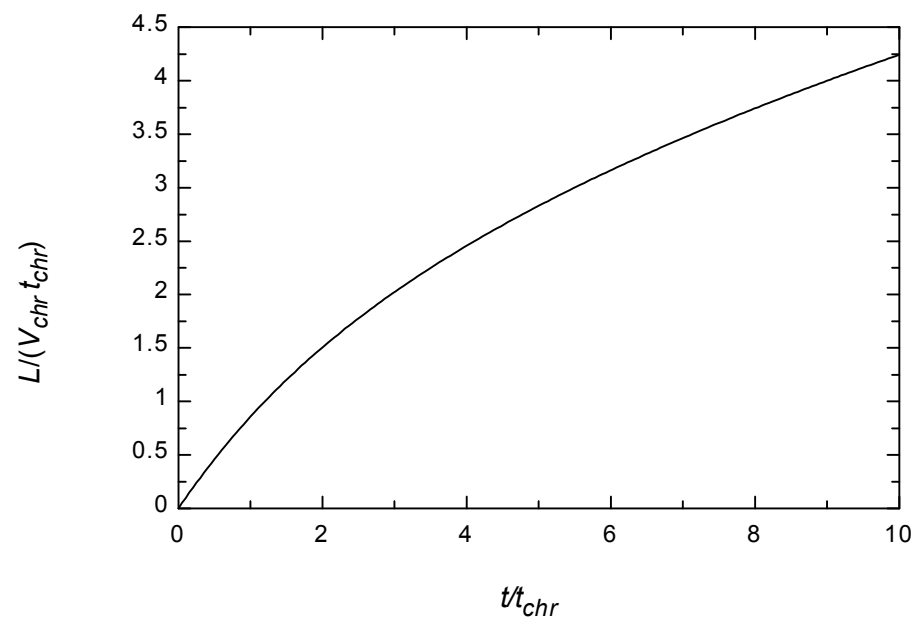

Figure 3: Variation of dimensionless penetration length as a function of dimensionless time.

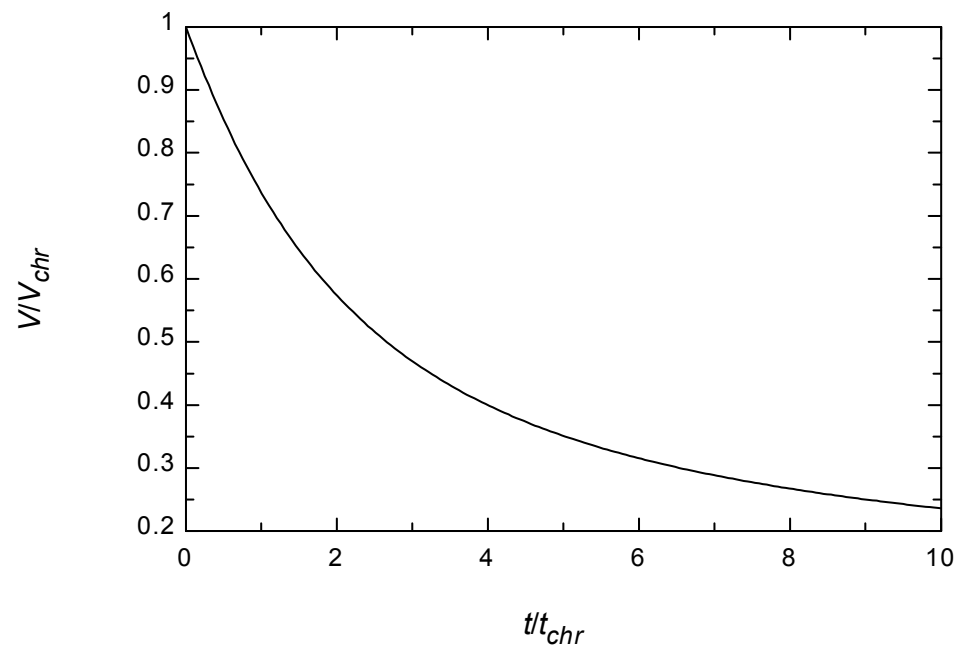

Figure 4: Variation of dimensionless velocity as a function of dimensionless time. 


\section{Comparisons with other models and experiments}

For the frictionless flow model, the flow velocity is constant and the distance travelled by the fluid in the capillary increases linearly with time. In the model including wall friction, the velocity decreases with time and the fluid penetrates smaller and smaller distances into the capillary.

Both Schwiebert and Leong [8] and Jong et al. [9] derived expressions for surface tension driven flow between parallel plates. In both studies the length as a function of time can be given as

$$
L=\left(\frac{a \sigma \cos \theta}{3 \eta} t\right)^{1 / 2},
$$

where $a$ is the separation distance between the plates. For parallel plates, the hydraulic diameter is $D_{h}=2 a$ and the constant appearing in the Fanning friction factor relation is $C=24$. This gives a characteristic velocity and characteristic time scale, respectively, of

$$
V_{c h r}=\left(\frac{2 \sigma \cos \theta}{\rho a}\right)^{1 / 2}
$$

and

$$
t_{c h r}=\frac{\rho a^{2}}{12 \eta} .
$$

Based on the material property values given in [8] and. [9], the time scales are on the order of $5 \times 10^{-6} \mathrm{~s}$, validating the use of the large time approximation of Eq. (31). Substituting Eqs. (34) and (35) into Eq. (31) yields

$$
L=\left(\frac{a \sigma \cos \theta}{3 \eta} t\right)^{1 / 2},
$$

which is identical to Eq. (33). As [8] and [9] both showed good agreement of Eq. (33) with experimental values of $L$, this serves as validation of the present model for times larger than the characteristic time scale $t_{c h r}$.

For systems with larger hydraulic diameters, large fluid densities and/or small viscosities, however, the characteristic time scale may become a significant fraction of a second. As such, the approximations of Eqs. (31) and (32) to which previous models reduce may show significant deviations from measured values. This is especially evident in Eq. (32) in which the velocity approaches infinity as $t \rightarrow 0$. Eq. (30), however, predicts $V=V_{c h r}$ at $t=0$, likely indicating a better model for small times and/or systems with large characteristic time scales. Physically this value makes sense as well, as $V_{c h r}$ represents a velocity characterized by zero wall friction. Since the fluid in the capillary initially makes contact with the wall over an infinitesimally small surface area, it should approximate frictionless flow at $t=0$. However, experimental values for velocity are needed as evidence for this claim. Further study is therefore warranted. 


\section{Conclusion}

Models for surface tension driven flow in a capillary of arbitrary cross section have been developed using macroscopic conservation equations. Models for flow with and without wall friction were both investigated. For flow without friction, the flow velocity is constant and is a function of surface tension, contact angle, fluid density, and hydraulic diameter. For flows with wall friction, a characteristic time for the flow can be calculated. For flow times on the order the characteristic time scale, the flow velocity decays exponentially from the frictionless flow velocity. For larger times, both velocity and penetration length show a power law dependence on time.

For larger time scales, the derived equations for length and velocity reduce to expressions developed by earlier models. Previously obtained experimental values of penetration length validate the present model. However, experimentally measured values of flow velocity are needed to validate the model's accuracy for small times or systems with large characteristic time scales.

\section{Acknowledgements}

The authors wish to thank Drs. L. Olson and C. Lui for their generous comments and suggestions in regard to this work.

\section{References}

[1] Londe, G., Wesser, A., Cho, H.J., Zhai, L., Chunder, A. \& Subbarao, S., Passive microfluidic valve using superhydrophobic/hydrophyilic nanostructures for lab-on-a-chip (LOC) systems. Proc. 14 ${ }^{\text {th }}$ Annual international conference on solid state sensors, actuators and Microsystems (Transducers '07), Lyon, pp. 1801-1804, June 10-14, 2007.

[2] Feng, Y., Zhou, Z., Ye, X., \& Xiong, J., Passive valves based on hydrophobic microfluidics, Sensors and Actuators A 108, pp 138-143, 2003.

[3] Vanifatova, N.G., Zavarzina, A.G., \& Spivakov, B.Y., Potential of capillary zone electrophoresis for estimation of humate acid-base properties, $J$. Chromatography A, 1183(1-2, 7 pp 186-191, March 2008.

[4] Macka, M., Andersson, P., \& Haddad, P.R., Changes in Electrolyte pH Due to Electrolysis during Capillary Zone Electrophoresis. Anal. Chem., 70(743), 1998.

[5] T. Young, Miscellaneous Works, ed G. Peacock J., Murray: London, p. 418, 1885.

[6] Lucas, R., Kolloid Z., 23(15), 1918.

[7] Washburn, E. W., The dynamics of capillary flow, Phys. Rev., 17(273), pp 273-283, 1921.

[8] Schwiebert, M.K. \& Leong, W.H., IEEE Trans. Compon. Packag. Manuf. Technol. 19(2), pp 133-137, 1996.

[9] Jong, W.R., Kuo, T.H., Ho, S.W., Chiu, H.H. \& Peng, S.H., Flows in rectangular microchannels driven by capillary, Int. Comm. in Heat and Mass Trans., 34, pp 186-196, 2007. 